\title{
Relative Magnitudes of Geomagnetic Daily-Range Measures near the Dip Equator
}

\author{
A. ONWUMEChilli* and P.O. OgBuehi* \\ Department of Physics, University of Ibadan, Nigeria.
}

(Received November 4, 1966)

\begin{abstract}
Different measures of range in use are defined and discussed. Expressions are obtained (a) for correcting the smoothing effects on the various measures of range, and (b) for comparing two measures of range. It is shown how these depend on the time of maximum and therefore to some extent on season. The smoothing corrections when the maximum is at $1100 \mathrm{hrs}$. L.T. vary from $\frac{1}{2}$ to $15 \frac{1}{2} \%$ for measures of range based on averaging over one to 4 hours. The ratio of any two measures of range is found not to be significantly different between $H$ at Huancayo and $Z$ at Ibadan nor between years of solar activity maximum and minimum.
\end{abstract}

\section{Introduction}

Many anlyses of geomagnetism are based on the daily range, $M$, of magnetic elements. Investigators of equatorial geomagnetic variations have used different measures of daily range. This has made comparison of their results difficult. Our object is to examine these various measures of range with a view to establishing a method of comparing them quantitatively. This would remove the difficulty in discussing the results of different workers.

\section{Measures of Range}

Although the word range means maximum minus minimum, instantaneous values should never be used in obtaining range because the geomagnetic field is very variable especially near the dip equator where short duration fluctuations are highly amplified (Onwumechilli 1959, Onwumechilli and Ogbuehi 1962). The following definitions of range have been used by electrojet investigators (arranged in our order of preference)

$$
\begin{aligned}
& M_{4}=\frac{1}{4}\left(H_{10}+H_{11}+H_{12}+H_{13}\right)-\frac{1}{4}\left(H_{00}+H_{01}+H_{22}+H_{23}\right) . \\
& M_{2}=\frac{1}{2}\left(H_{11}+H_{12}\right)-\frac{1}{4}\left(H_{00}+H_{01}+H_{22}+H_{23}\right) . \\
& M_{3}=\frac{1}{3}\left(H_{10}+H_{11}+H_{12}\right)-\frac{1}{4}\left(H_{00}+H_{01}+H_{22}+H_{23}\right) . \\
& M_{1}=H_{\text {max. }}-\frac{1}{4}\left(H_{00}+H_{01}+H_{22}+H_{23}\right) . \\
& M_{m}=H_{\text {max. }}-H_{\text {min. }} .
\end{aligned}
$$

* Now at Department of Physics, University of Nigeria, Nsukka, Eastern Nigeria. 
where $H_{t}$ is usually the average value of the element under study from the hour $t$ to the hour $(t+1)$ local time. $M_{T}$ is the average of $H$ over $T$ hours less the midnight average of $H$. It would make little difference if the mignight average is taken as $\frac{1}{2}\left(H_{00}+H_{23}\right)$. Midnight

Table 1 Monthly values of the departure $M_{4}$, and the ratios, $R_{T_{4}}$, of other departures to it. $H r$ is the hour of maximum.

\begin{tabular}{|c|c|c|c|c|c|c|c|c|c|c|c|c|}
\hline & JAN & FEB & MAR & APR & MAY & JUN & JUL & AUG & SEP & OCT & NOV & DEC \\
\hline \multicolumn{13}{|c|}{ Huancayo $H 1954$} \\
\hline$M_{4}$ & 80.5 & 89.0 & 91.8 & 82.5 & 93.2 & 52.8 & 61.7 & 64.3 & 100.5 & 118.7 & 52.5 & 66.5 \\
\hline$R_{14}$ & 1.158 & 1.081 & 1.144 & 1.133 & 1.080 & 1.061 & 1.122 & 1.096 & 1.139 & 1.147 & 1.434 & 1.191 \\
\hline$R_{24}$ & & 1.081 & 1.058 & 1.091 & 1.064 & 1.061 & 1.041 & 1.058 & 1.095 & 1.072 & 1.042 & 1.101 \\
\hline$R_{34}$ & 1.096 & 1.036 & 1.086 & 1.048 & 1.034 & 1.023 & 1.068 & 1.039 & 1.087 & 1.086 & 1.171 & 1.090 \\
\hline$R_{m_{4}}$ & 1.180 & 1.146 & 1.155 & 1.200 & 1.116 & 1.136 & 1.135 & 1.120 & 1.154 & 1.163 & 1.448 & 1.278 \\
\hline$H r$ & 10,11 & 11,12 & 10 & 11 & 11 & 11,12 & 10 & 11 & 11 & 11 & 09,10 & 11 \\
\hline
\end{tabular}

Huancayo $H 1958$

\begin{tabular}{c|c|c|c|c|c|c|c|c|c|c|c|c}
\hline$M_{4}$ & 153.9 & 186.6 & 199.8 & 183.0 & 157.5 & 164.0 & 152.8 & 148.5 & 218.8 & 202.0 & 132.3 & 159.5 \\
\hline$R_{14}$ & 1.226 & 1.070 & 1.106 & 1.079 & 1.070 & 1.111 & 1.080 & 1.104 & 1.058 & 1.119 & 1.122 & 1.182 \\
\hline$R_{24}$ & 1.086 & 1.061 & 1.081 & 1.068 & 1.063 & 1.059 & 1.063 & 1.071 & 1.053 & 1.069 & 1.028 & 1.069 \\
\hline$R_{34}$ & 1.132 & 1.023 & 1.020 & 1.026 & 1.025 & 1.046 & 1.010 & 1.035 & 1.023 & 1.064 & 1.060 & 1.094 \\
\hline$R_{m 4}$ & 1.268 & 1.082 & 1.143 & 1.093 & 1.079 & 1.122 & 1.111 & 1.111 & 1.064 & 1.124 & 1.172 & 1.235 \\
\hline$H r$ & 10,11 & 12 & 11 & 11 & 11 & 11 & 12 & 11 & 11 & 11 & 10 & 11 \\
\hline
\end{tabular}

Ibadan $Z 1958$

\begin{tabular}{c|c|c|c|c|c|c|c|c|c|c|c|c}
\hline$M_{4}$ & 47.5 & 41.4 & 40.5 & 52.2 & 51.5 & 36.5 & 34.3 & 47.1 & 47.2 & 39.5 & 39.1 & 36.3 \\
\hline$R_{14}$ & 1.168 & 1.360 & 1.217 & 1.088 & 1.142 & 1.099 & 1.160 & 1.113 & 1.112 & 1.385 & 1.212 & 1.187 \\
\hline$R_{24}$ & 1.149 & 1.114 & 1.042 & 1.042 & 1.140 & 1.088 & 1.090 & 1.104 & 1.046 & 1.023 & 1.031 & 1.011 \\
\hline$R_{34}$ & 1.059 & 1.196 & 1.101 & 1.056 & 1.047 & 1.016 & 0.977 & 0.998 & 1.042 & 1.144 & 1.092 & 1.069 \\
\hline$R_{m 4}$ & 1.200 & 1.400 & 1.472 & 1.280 & 1.363 & 1.288 & 1.280 & 1.448 & 1.390 & 1.499 & 1.253 & 1.289 \\
\hline$H r$ & 12 & 10 & 10 & 11 & 12 & 12 & 12 & 12 & 11 & 10 & 10 & 10 \\
\hline
\end{tabular}

Ibadan $Z 1964$

\begin{tabular}{c|c|c|c|c|c|c|c|c|c|c|c|c}
\hline$M_{4}$ & 15.5 & 25.3 & 29.4 & 21.3 & 13.2 & 14.8 & 15.3 & 20.6 & 22.1 & 20.5 & 31.8 & 28.9 \\
\hline$R_{14}$ & 1.368 & 1.154 & 1.190 & 1.127 & 1.098 & 1.243 & 1.144 & 1.136 & 1.318 & 1.556 & 1.453 & 1.017 \\
\hline$R_{24}$ & 0.974 & 1.103 & 1.058 & 1.075 & 1.091 & 1.128 & 1.118 & 1.073 & 1.041 & 1.029 & 1.057 & 1.045 \\
\hline$R_{34}$ & 1.103 & 1.055 & 1.099 & 1.094 & 1.045 & 1.128 & 1.105 & 1.049 & 1.131 & 1.205 & 1.198 & 1.014 \\
\hline $\mathrm{R}_{m 4}$ & 1.390 & 1.344 & 1.347 & 1.371 & 1.136 & 1.297 & 1.647 & 1.641 & 1.511 & 1.561 & 1.516 & 1.073 \\
\hline$H r$ & 09 & 11 & 10 & 10 & 12 & 11 & 11 & 11 & 10 & 10 & 10 & 12 \\
\hline
\end{tabular}


value is taken as a base because of the quiescence of the Sq currents then.

The more the hourly values averaged, the more stable is the range, thus $M_{m}$ and $M_{1}$ are more variable than the rest. They also embody two difficulties that can be helped. The hour of maximum or minimum of any element varies from day to day and from place to place. Thus if they are used for comparing many days or many places they do not give the state of the field at any one time. Furthermore $M_{1}$ and $M_{m}$ are always positive by definition and fail to give any indication of the direction of change. Obviously $M_{4}$ and $M_{3}$ are the least susceptible to disturbance and minor fluctuations.

Two of the advantages of fixing the hours for calculating $M_{2}, M_{3}$ and $M_{4}$ are: (a) they give the state of the field at the same local time everyday and everywhere; and (b) they give the additional information of the direction of change for example their sign at once indicates whether the element is greater near mid-day than midnight and if the daily swing is reversed on opposite sides of the current focus or the equator. Strictly speaking, they are better termed departures or differences or magnitudes.

It is of interest to obtain the relations between the departures, $M_{T},(T=1$ to 4$)$ as well as the relation between them and the maximum departure for the day in order to be able to compare the results of different investigators. We define departure ratios

$$
R_{T 4}=\frac{M_{T}}{M_{4}}
$$

where $T=1$ to 3 .

\section{Two ways of computing mean range}

Here we compare the mean ranges obtained by the two methods usually used. In method I the hourly values are first averaged for all the days of interest before taking the range or departure. In method II ranges for individual days are averaged.

Mean values of $M_{T}$, ( $T=1$ to 4 ) were computed by the two methods using the 5 international quiet days in each month. Altogether 24 monthly means of $M_{T}$ were obtained for $H$ at Huancayo. The values of $M_{2}$ calculated by the two methods did not differ by up to one gamma in any of the 24 months. The same was true of $M_{3}$ and $M_{4}$.

On the other hand, in 23 of the 24 months, method II gave values of $M_{1}$ and $M_{m}$ greater by 1 to 7 gammas than their values by method I. The proportional difference will be appre-

Table 2 Annual means of the departure $M_{4}$, and the ratios, $R_{T_{4}}$, of other departures to it.

\begin{tabular}{l|c|c|c|c}
\hline & $\begin{array}{c}\text { Huancayo } \\
H\end{array}$ & $\begin{array}{c}\text { Huancayo } \\
H\end{array}$ & $\begin{array}{c}\text { Ibadan } \\
Z\end{array}$ & $\begin{array}{c}\text { Ibadan } \\
Z \\
\end{array}$ \\
& 1954 & 1958 & 1958 & 1964 \\
\hline$M_{4}$ & 79.5 & 171.6 & 42.7 & 61.6 \\
$R_{14}$ & $1.149 \pm 0.027$ & $1.111 \pm 0.012$ & $1.187 \pm 0.027$ & $1.234 \pm 0.044$ \\
$R_{24}$ & $1.069 \pm 0.006$ & $1.066 \pm 0.006$ & $1.073 \pm 0.014$ & $1.066 \pm 0.012$ \\
$R_{34}$ & $1.072 \pm 0.011$ & $1.047 \pm 0.007$ & $1.066 \pm 0.018$ & $1.101 \pm 0.017$ \\
$R_{m 4}$ & $1.186 \pm 0.026$ & $1.134 \pm 0.016$ & $1.347 \pm 0.237$ & $1.403 \pm 0.050$ \\
Count & 12 & 12 & 12 & 12 \\
\hline
\end{tabular}


ciated when the following annual mean values of $R_{14}$ and $R_{m 4}$ obtained by method II are compared with their corresponding values by method I in Table 2 .

Values of $R_{14}$ and $R_{m 4}$ obtained by Method II, using Huancayo $H$.

\begin{tabular}{c|c|c}
\hline & 1954 & 1958 \\
\hline$R_{14}$ & $1.19 \pm 0.03$ & $1.14 \pm 0.01$ \\
$\mathrm{R}_{m_{4}}$ & $1.27 \pm 0.04$ & $1.18 \pm 0.02$ \\
\hline
\end{tabular}

$Z$ at Ibadan in January, April, and June 1958 was also tested as above. The results were found to be similar to those of $H$ at Huancayo.

It may therefore be concluded that the mean values of the fixed hour departures, $M_{2}$, $M_{3}$, and $M_{4}$ for international quiet days are the same whichever method of computation is used. On the other hand, method II usually gives a slightly higher average of $M_{1}$ and $M_{m}$ respectively than method I. This represents a further advantage in using the fixed hour departures.

All the values given in succeeding sections are obtained by method I.

\section{Theoretical approximation}

We approximate the portion of the daily variation curve near the maximum to the cosine function of time

$$
H=H_{0} \cos w t
$$

where $w$ is an angle, $H_{0}$ is maximum $H$, and $t$ is time measured from the position of maximum $H$. The mean value of $H$ from

$$
\begin{aligned}
t & =t_{0} \text { to } t=t_{0}+T \text { is } \\
M_{T}\left(t_{0}\right) & =\frac{1}{T} \int_{t_{0}}^{t_{0}+T} H(t) d t \\
& =\frac{H_{0}}{w T}\left[\sin w\left(t_{0}+T\right)-\sin w t_{0}\right] .
\end{aligned}
$$

This enables us to correct the departure $M_{T}\left(t_{0}\right)$ for smoothing. The smoothing correction is

$$
C_{T}\left(t_{0}\right)=\frac{H_{0}}{M_{T}\left(t_{0}\right)}=\frac{w T}{\sin w\left(t_{0}+T\right)-\sin w t_{0}} .
$$

In this expression, one should really write $t_{0 T}$ since $t_{0}$ is different for each $M_{T}$. It follows that the departure ratios, Equation (6) are given by

$$
R_{T_{4}}=\frac{C_{4}}{C_{T}}=\frac{4}{T} \frac{\left[\sin w\left(t_{0 T}+T\right)-\sin w t_{0 T}\right]}{\left[\sin w\left(t_{04}+4\right)-\sin w t_{04}\right]} .
$$

Because the initial time, $t_{0}$ for $M_{2}$ is always one hour greater than the initial time for $M_{4}$,

$$
R_{24}=\sec w .
$$

For any given hour of maximum it is possible to express $R_{T 4}$ in terms of $R_{24}$. Such relationships permit a test of the usefulness of the approximation before application. 


\section{Numerical values of the corrections}

Tables 1 and 2 show numerical values calculated from $H$ at Huancayo and $Z$ at Ibadan for international quiet days during years of sunspot maximum 1958 and minimum 1954 and 1964. There is no striking difference between years of high and low solar activity nor between $H$ and $Z$ except those differences arising from the dependence of the ratio on time of maximum. For example Fig. 1 shows some seasonal effect on $R_{14}$ and $R_{m 4}$ for $Z$ at Ibadan in 1958 arising from the seasonal variation of the time of maximum. No such variation is obvious in the ratios $R_{T}(H)$ where the time of maximum is more uniform.

Table 3 shows the average of $R_{r 4}$ according to hours of maximum. As the hour of maximum advances from 1000 to $1200, R_{14}, R_{34}$, and $R_{m 4}$ decrease but $R_{24}$ increases. To test the usefulness of the theoretical approximation we use the mean $R_{24}$ in Table 3 to calculate $R_{14}$ and $R_{34}$, assuming that in the case of $M_{1}$ the maximum is at the beginning of the interval.

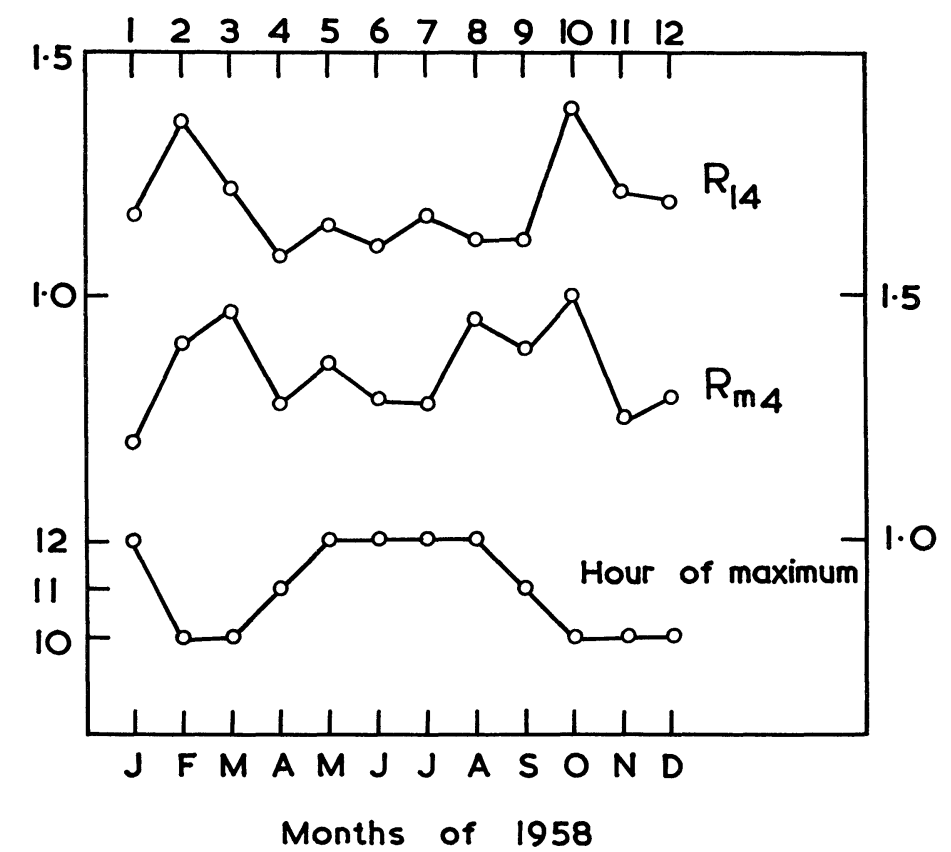

Figure 1. Variations of the departure ratios, $R_{14}$ and $R_{m_{4}}$, for $Z$ at Ibadan in 1958 arising from the variation in the time of maximum.

Table 3 Mean values of departure ratios, $R_{T_{4}}$, when maximum occurs at 1000,1100 , and 1200 hours local time respectively.

\begin{tabular}{l|c|c|c|c}
\hline Hour & 1000 & 1100 & 1200 & All \\
\hline$R_{14}$ & $1.261 \pm 0.038$ & $1.125 \pm 0.009$ & $1.105 \pm 0.016$ & $1.170 \pm 0.016$ \\
$R_{24}$ & $1.047 \pm 0.006$ & $1.076 \pm 0.006$ & $1.092 \pm 0.013$ & $1.068 \pm 0.005$ \\
$R_{34}$ & $1.118 \pm 0.014$ & $1.058 \pm 0.005$ & $1.021 \pm 0.008$ & $1.072 \pm 0.007$ \\
$R_{m 4}$ & $1.360 \pm 0.040$ & $1.230 \pm 0.037$ & $1.220 \pm 0.042$ & $1.267 \pm 0.023$ \\
Count & 13 & 20 & 9 & 48 \\
\hline
\end{tabular}


The results are as follows.

\begin{tabular}{l|r|r|r|r|r|r}
\hline \multirow{2}{*}{} & \multicolumn{2}{|c|}{1000} & \multicolumn{2}{c|}{1100} & \multicolumn{2}{c}{1200} \\
\cline { 2 - 7 } & $R_{14}$ & $R_{34}$ & $R_{14}$ & $R_{34}$ & $R_{14}$ & \multicolumn{1}{c}{$R_{34}$} \\
\hline Calculated & 1.27 & 1.12 & 1.16 & 1.10 & 1.09 & 1.03 \\
Observed & 1.26 & 1.12 & 1.13 & 1.06 & 1.11 & 1.02 \\
Error Limits & \pm 0.12 & \pm 0.05 & \pm 0.04 & \pm 0.02 & \pm 0.05 & \pm 0.02 \\
\hline
\end{tabular}

The error limits indicate the range within which the difference between the calculated and observed ratios must lie so that the difference will not be satistically significant at $5 \%$ level. Thus only one of the six calculated ration is significantly different from its observed mean at $5 \%$ level. We may therefore say with that much confidence that the theoretical approximation is useful. $w$ depends on time of maximum but from the overall average of $R_{24}=1.068$ from Huancayo $H$ and Ibadan $Z$ we infer from Equation (11) that $w=20.55^{\circ}$, approximately $\frac{\pi}{9}$. The difference between this and the value for 1100 is within error limits.

Assuming the maximum is at $1100 \mathrm{hrs}$. L.T. and taking $w=\frac{\pi}{9}$ we proceed to calculate the smoothing corrections $C_{T}$, from Equation (9) to be applied to the departures, $M_{T}$, Equations (1) to (4)

$$
\begin{aligned}
& C_{1}=\frac{1}{2} w \operatorname{cosec} \frac{1}{2} w=1.005 \\
& C_{1}=w \operatorname{cosec} w=1.020 \\
& C_{2}=w \operatorname{cosec} w \sec w=1.085 \\
& C_{3}=\frac{3 w}{\sin 2 w+\sin w}=1.063 \\
& C_{4}=w \operatorname{cosec} w \sec ^{2} w=1.155
\end{aligned}
$$

Equation (13) applies when the maximum is at one end of the hour interval and Equation (12) when the hourly mean is centred on the time of maximum. The midnight period used as zero level is usually flat and needs no smoothing correction. $C_{1}$ applies to the $H_{\max }$. in $M_{m}$ but the theory does not cover the hour of minimum during which the shape may not be approximately a cosine function. These smoothing corrections should be specifically determined for each investigation using Equation (11), but if that is not possible for any reason the numerical values in Equations (12) to (16) may be taken as order of magnitude values.

\section{Conclusion}

Having studied the various measures of range used in equatorial geomagnetism we find that:

1. The fixed hour departures, $M_{2}, M_{3}$, and $M_{4}$, are not affected by the method of computing mean for international quiet days. The measures involving maximum hourly mean, $M_{1}$, and $\mathrm{M}_{m}$, are affected by the method of computing mean because the time of maximum varies. from day to day. 
2. The annual mean ratios of all the other measures of range to $M_{4}$ change monotonically as the hour of maximum changes from 1000 to 1200 L.T. and this is not merely because of the definition of $M_{4}$ for it is also true of the ratio of $M_{m}$ to $M_{1}$.

3. The expressions developed for calculating smoothing correction factors, $C_{T}$, are appropriate except in the case of the orthodox measure of range $M_{m}$. The difficulties with $M_{m}$ are that the time of minimum changes and also the magnetogram in the region of minimum is not like the mathematical representation.

4. The smoothing correction factors lead to expressions for converting one measure of range into another. The conversion ratios have been found suitable except when the orthodox measure of range $\mathrm{M}_{m}$ is involved.

5. The annual means of the conversion ratios for $H$ at Huancayo are not noticeably different from those for $Z$ at Ibadan nor are they different for years of high and low solar activity.

It is clear from the above that the measures of range based on fixed hour departures are more regular and tractable than the orthodox measure of range. Because of this and two other advantages mentioned in section 2 we prefer the use of the fixed hour departures to the orthodox measure of range. Furthermore, since the departures can now be corrected for smoothing we prefer the most stable $M_{4}$ to the others.

\section{Acknowledgement}

This work has been supported with our I.Q.S.Y. grant for which we are grateful to the Federal Government of Nigeria.

\section{References}

Onwumechilli, C.A. The Relation between $H$ - and $Z$-Variations near the equatorial electrojet. J. Atmosph. Terr. Phys. 16, 274-282, 1959.

Onwumechilli, A., and Ogbuehi, P.O. Fluctuations in the geomagnetic horizontal field. J. Atmosph. Terr. Phys. 42, 173-190, 1962. 